\title{
Erratum to: Radical resection of nongerminomatous pineal region tumors via the occipital transtentorial approach based on arachnoidal consideration: experience on a series of 143 patients
}

\author{
Songtao Qi • Jun Fan • Xi-an Zhang • Hui Zhang • \\ Binghui Qiu • Luxiong Fang
}

Received: 5 August 2014 / Accepted: 24 October 2014 / Published online: 10 December 2014

(C) Springer-Verlag Wien 2014

Erratum to: Acta Neurochir (2014) 156:2253-2262

DOI 10.1007/s00701-014-2224-4

The original version of this article unfortunately missed to publish the comments from Dr. Amir Dehdashti, which is the below:

This is a retrospective review of 143 patients with pineal region tumors (non germinoma) who underwent resection via occipital interhemispheric transtentorial approach. The follow-up on 130 patients shows an overall good outcome, $18 \%$ complication and a significant mortality of $8.5 \%$.

There is nothing novel in this article although the experience is impressive. The description of arachnoid layers is easily explained by the nature and location of the tumor whether coming directly from the pineal gland (NGGCT and pineal tumors) or others outside the arachnoid layer (e.g. Meningioma). We would prefer to perform this procedure in park bench with the right side down allowing the occipital lobe to fall with gravity. As long as no major retraction is used and the occipital vein is thoroughly preserved, the resulting hemianopia will be temporary. This is our preferred approach for pineal region pathologies when there is some degree of hydrocephalus and if the tentorium has a steep angle.

Amir Dehdashti

NY, USA

The online version of the original article can be found at http://dx.doi.org/ 10.1007/s00701-014-2224-4.

S. Qi $(\bowtie) \cdot$ J. Fan $\cdot$ X.-a. Zhang $\cdot$ H. Zhang $\cdot$ B. Qiu $\cdot$ L. Fang Department of Neurosurgery, Nanfang Hospital, Southern Medical University, 1838 Guangzhou Dadao Bei Street, Guangzhou 510515, People's Republic of China

e-mail:nfsjwk@gmail.com 